\title{
Electric Field Ionization of High Rydberg States of Ar with Sequences of Identical Pulses
}

\author{
F. Merkt, R. J. Rednall, S. R. Mackenzie, and T. P. Softley \\ Physical and Theoretical Chemistry Laboratory, South Parks Road, Oxford OX1 3QZ, United Kingdom
} (Received 8 September 1995)

Novel field-ionization behavior of very high Rydberg states of $\operatorname{Ar}(n=50-250)$ is observed using sequential pulsed electric fields. Fast autoionization acts as a filter against adiabatic field ionization near the ${ }^{2} P_{1 / 2}$ threshold, whereas both diabatic and adiabatic field ionization occur near the ${ }^{2} P_{3 / 2}$ threshold. Sequences of up to 9 equivalent pulses yield recurrent field ionization signals near the ${ }^{2} P_{3 / 2}$ threshold, provided that the field returns close to zero between pulses, demonstrating a statistical redistribution of the Rydberg population amongst all states of a given Stark manifold near zero field. [S0031-9007(96)00126-3]

PACS numbers: 32.80.Rm, 32.30.Jc, 32.60.+i, 32.70.Fw

The behavior of very high Rydberg states (principal quantum number $n>100$ ) is not as well understood as that of states with low to intermediate quantum numbers $(n<50)$ for which extensive data are available $[1,2]$. It is found experimentally that there is a marked discontinuity between the properties of high Rydberg states and the lower- $n$ states. For instance, much longer lifetimes than expected from an $n^{3}$ scaling law are observed at very high $n$. The mechanisms leading to stabilization remain controversial (e.g., see Refs. $[3,4]$ ), and there is wide renewed interest in this area. We describe a new approach to investigate the properties of high Rydberg states, which is related to the methodology of selective field ionization (SFI) $([1,2]$ and references therein). Special attention is given to the response of high atomic Rydberg state populations to sequences of identical pulsed electric fields from which we derive important information on the nature of these states.

Very high Rydberg states are of importance to zerokinetic-energy (ZEKE) photoelectron spectroscopy and related techniques, which employ delayed pulsed field ionization (PFI) following photoexcitation. High Rydberg states are very sensitive to their environment, and, in general (including in the experiments described here), the delayed pulsed field probes a mixed population of Rydberg states which can differ significantly from the optically prepared population of low $l$, low $m$ states $[3,5]$. $l$ and $m$ mixing may occur as a result of inhomogeneous stray electric fields $[3,6]$ or collisions with neighboring particles [1-3,7-9]. Our ultimate goal is to assess the importance of these mechanisms from the analysis of the response of the Rydberg state population to pulsed electric fields. A previous study of argon in our laboratory had revealed that Rydberg states converging on the ${ }^{2} P_{1 / 2}$ threshold were abnormally long lived [9]. Of interest for the present studies is the contrasting behavior near the ${ }^{2} P_{1 / 2}$ and ${ }^{2} P_{3 / 2}$ thresholds in this system.

The field-ionization behavior of Rydberg states $n \leq 50$ has been well characterized with the help of Stark maps $[1,2]$ showing the energies of the states versus electric field. The nature of the field-ionization process depends on the energy path the atoms follow as the electric field grows. If all crossings are traversed diabatically, ionization occurs when the ionization rate exceeds a certain value characteristic of the experiment (typically $10^{8} \mathrm{~s}^{-1}$ ). The extreme redshifted state of the Stark manifold with principal quantum number $n$ ionizes at fields $\approx F /$ a.u. $=$ $1 / 9 n^{4}$, whereas the extreme blueshifted states ionize at fields approximately twice as large [1-3]. If all crossings are traversed adiabatically, ionization occurs in a narrow range of fields $F$ centered on $F /$ a.u. $=1 / 16 n^{4}$. LandauZener theory predicts that the adiabatic traversal becomes less likely at increasing values of $n$ and $m$ and at high field slew rates [10]. For the high Rydberg states considered here, a Stark map consists of a very dense network of overlapping manifolds of states, with many thousands of (avoided) crossings between zero field and ionization. It is the very large number of these crossings which makes the high Rydberg states qualitatively different from those previously studied, in addition to the mixed $m$ character under normal experimental conditions. Intermediate situations between diabatic and adiabatic field ionization ("nondiabatic") are likely to be important for high Rydberg states; although the probability for a given crossing to be traversed adiabatically is very small, the large number of crossings renders a nondiabatic process likely.

In the experiments described here, high Rydberg states of Ar are produced by single-photon excitation from the ground state $\left({ }^{1} S_{0}\right)$ using a coherent source of extreme ultraviolet radiation (see Ref. [5]) of bandwidth $1.5 \mathrm{~cm}^{-1}$, pulse length $10 \mathrm{~ns}$. Photoexcitation occurs in the presence of a weak stray field $(\sim 100 \mathrm{mV} / \mathrm{cm})$. An electric field pulse sequence is applied after a selected delay with respect to the laser, and the electrons produced by field ionization are directed along a mumetal-shielded timeof-flight (TOF) tube and are detected with an electron multiplier.

Figures 1(a) and 1(b) show delayed PFI spectra of Rydberg states converging on the ${ }^{2} P_{3 / 2}$ and the ${ }^{2} P_{1 / 2}$ thresholds, respectively, for various field amplitudes. These 


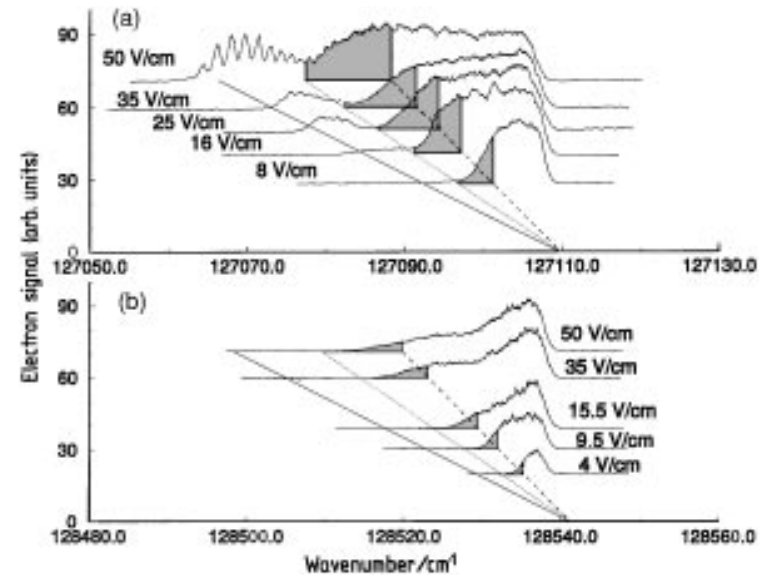

FIG. 1. Delayed PFI spectra of Rydberg states converging on (a) the ${ }^{2} P_{3 / 2}$ and (b) ${ }^{2} P_{1 / 2}$ thresholds of $\mathrm{Ar}$, for various amplitudes of the single pulsed field (rise time $100 \mathrm{~ns}, 1 \mu \mathrm{s}$ delay with respect to excitation). The vertical position of the baselines is scaled as $F^{1 / 2}$. The expected low frequency onsets for adiabatic field ionization (full line) and for the diabatic field ionization of the most redshifted (dotted line) and the most blueshifted (dashed line) Stark states are shown. The region between the two diabatic onsets is shaded.

spectra were obtained by applying a single pulsed field (rise time $100 \mathrm{~ns}$ ) $1 \mu \mathrm{s}$ after photoexcitation. Also indicated in Fig. 1 are the expected low frequency onsets for adiabatic field ionization (full lines), and for the diabatic field ionization of the most redshifted (dotted lines) and the most blueshifted (dashed lines) Stark states assuming a minimum ionization rate of $10^{8} \mathrm{~s}^{-1}$; the region between the two diabatic onsets is shaded. Both adiabatic/ nondiabatic and diabatic field ionization are observed for Rydberg states below the ${ }^{2} P_{3 / 2}$ threshold [Fig. 1(a)]. The adiabatic/nondiabatic field-ionization signal appears as a weak tail on the red side of the strong diabatic field-ionization peak at the lowest fields, but its importance grows rapidly as the magnitude of the pulsed field increases (thereby enabling successively lower Rydberg states to be field ionized). The importance of adiabatic/ nondiabatic field ionization is greatly reduced at the ${ }^{2} P_{1 / 2}$ threshold [see Fig. 1(b)] where the field-ionization signals occur exclusively in the range where diabatic field ionization is expected.

For a Rydberg state to ionize at a lower field than predicted for diabatic field ionization, as in Fig. 1(a), at least one avoided crossing with another Stark state needs to be traversed adiabatically in the energy region above the classical saddle point potential. At the crossing, the atom changes its quantum state, a process that can only occur when the electron passes close to the core region. An electron in a Rydberg state with a ${ }^{2} P_{1 / 2}$ core above the ${ }^{2} P_{3 / 2}$ threshold will be rapidly ejected by spin-orbit autoionization if it can approach the core region: Hence, the states that would undergo nondiabatic/adiabatic fieldionization decay instead by spin-orbit autoionization be- fore the pulsed field is applied and do not contribute to the PFI spectra. The reverse is also true: A Rydberg state that survives the delay time without undergoing spinorbit autoionization must have a vanishing probability of returning to the core region, and therefore field ionizes diabatically as is observed experimentally. Spin-orbit autoionization is thus demonstrated to be a filter against nondiabatic/adiabatic field ionization. This new finding is expected to be perfectly general and important in the context of ZEKE/PFI spectroscopy; any rapid internal decay process in atomic and molecular Rydberg states such as autoionization and/or predissociation will act as an effective filter against nondiabatic/adiabatic field ionization.

Figure 2 illustrates the effect of two sequential equivalent pulsed fields (rise time $70 \mathrm{~ns}$, magnitude $10 \mathrm{~V} / \mathrm{cm}$, separation $3 \mu \mathrm{s}$ ) on Rydberg states below the ${ }^{2} P_{3 / 2}$ threshold. Electron TOF profiles are shown for different excitation wave numbers. The stray field in our apparatus $(\sim 100 \mathrm{mV} / \mathrm{cm})$ prevents the observation of the highest Rydberg states in Fig. 2. The experiments represented by Figs. 2(a) and 2(b) differ in that the electric field returns to $0 \mathrm{~V} / \mathrm{cm}$ in between the pulses in the case of

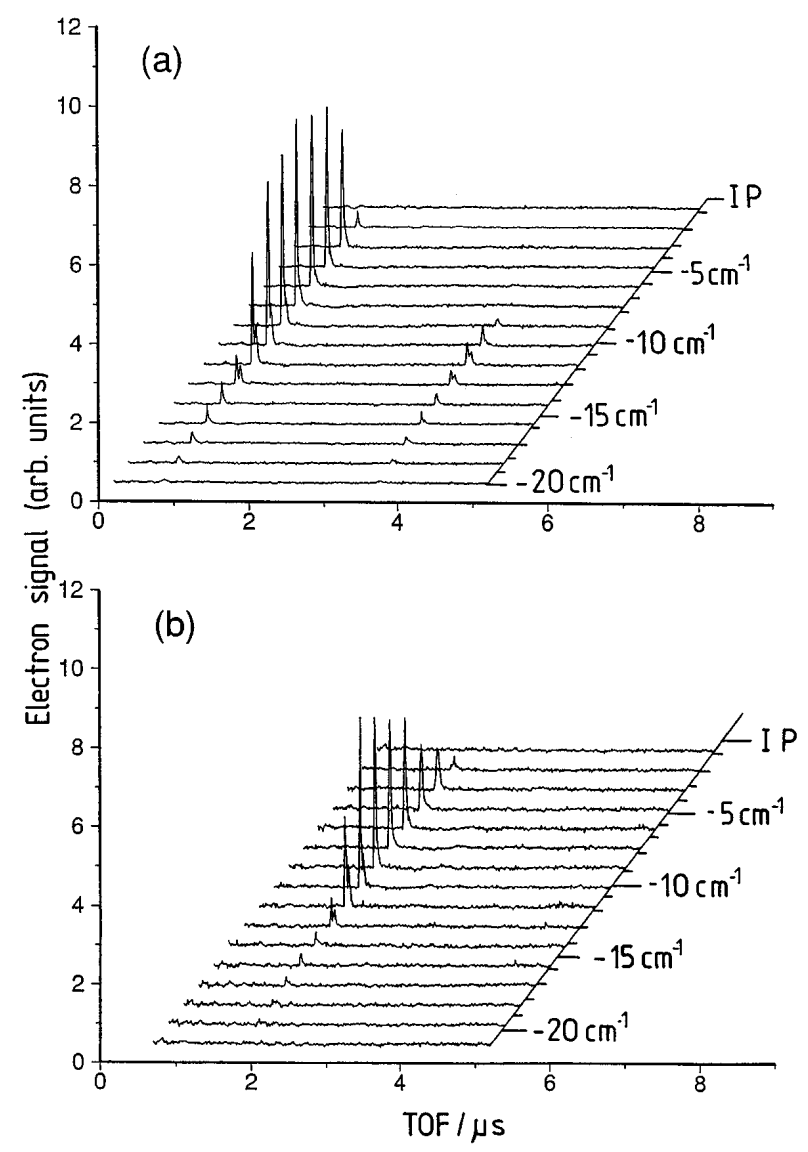

FIG. 2. Effect of two equivalent pulsed fields (rise time 70 $\mathrm{ns}$, magnitude $10 \mathrm{~V} / \mathrm{cm}$, separation $3 \mu \mathrm{s}$ ) on Rydberg states below the ${ }^{2} P_{3 / 2}$ threshold. Electron TOF profiles are shown at different energies with respect to threshold (IP), indicated along the $y$ axis. The electric field returns to (a) $0 \mathrm{~V} / \mathrm{cm}$ and (b) 0.75 $\mathrm{V} / \mathrm{cm}$ in the interval separating the pulses. 
Fig. 2(a) but only to $0.75 \mathrm{~V} / \mathrm{cm}$ in Fig. 2(b). Whereas only the first pulsed field contributes to the ionization signal in Fig. 2(b), a second peak grows in Fig. 2(a) in the region below $n=120$ where the first peak starts to drop in intensity, i.e., in the diabatic fall-off range $\approx-3.0 \mathrm{~cm}^{-1} \sqrt{F / \mathrm{Vcm}^{-1}}$ to $-4.6 \mathrm{~cm}^{-1} \sqrt{F / \mathrm{Vcm}^{-1}}$. Clearly some of the Rydberg atoms not ionized by the first pulse are ionized by the second identical pulse. The second peak continues into the region below $\approx-4.6 \mathrm{~cm}^{-1} \sqrt{F / \mathrm{Vcm}^{-1}}$ where the field ionization must be nondiabatic. The application of the same pulse sequences at the ${ }^{2} P_{1 / 2}$ limit reveals essentially the same results as shown in Fig. 2, although the range of partial ionization is much narrower and confined to the diabatic fall-off region shaded in Fig. 1(b).

In the diabatic fall-off region, the atoms contributing to the second field-ionization peak must be those that were in states blueshifted by the first field, because such states have the lowest diabatic ionization rates [1,2]. However, the observation of a second signal implies that at least some of the blueshifted Stark states have been converted to redshifted states in the interval separating the two pulsed fields: a change has occurred in the parabolic quantum numbers $n_{1}$ and $n_{2}$ such that $k=n_{2}-n_{1}$ has increased. Moreover, the absence of the second peak in Fig. 2(b) demonstrates that this change in $k$ can only take place once the field has been lowered below 0.75 $\mathrm{V} / \mathrm{cm}$. This explanation is not sufficient to account for the absence of a second signal in the nondiabatic region when the field does not return to zero: in this case we believe that $m$-changing processes also occur near zero field, converting high- $m$ states, resistant to adiabatic crossings, into low- $m$ states which can be efficiently ionized nondiabatically.

When a sequence of three identical pulses is used, it is found that a third electron signal can be observed, provided that the field returns to zero between each pulse. However, if the field is reduced only to 0.75 $\mathrm{V} / \mathrm{cm}$ between pulses 1 and 2 and then to zero between pulses 2 and 3, then an electron signal is obtained from pulses 1 and 3 only; the signal from pulse 3 is the same magnitude as the signal from pulse 2 when the field is zero between pulses 1 and 2 . This demonstrates that the condition for the observation of an ionization signal from any pulsed field apart from the first is that the field returns sufficiently close to zero before its application. In practice, we observe a rapid decrease of the signal caused by the second pulsed field as the magnitude of the intermediate field is increased from 0 to $0.5 \mathrm{~V} / \mathrm{cm}$. This phenomenon is strongly related to that observed by Lee et al. [11] in a study of ionization of $\mathrm{Na}$ in combined microwave and static fields, where a periodic return to zero field was also required.

The results reported above suggest that in the diabatic fall-off region, and when the electric field is brought close to zero, the Rydberg states lose memory, at least partially, of the parabolic quantum numbers $n_{1}$ and $n_{2}$ that characterized their behavior during the previous pulsed field. In the limit of complete loss of memory, a pulsed field will probe the part of the initial population of Rydberg states that has not yet been ionized by previous pulses and that has been randomly distributed among all accessible Stark states. Each successive pulse thus probes the same statistical distribution of Stark states and ought to have the same energy-dependent fieldionization efficiency $X(n)$ [with $0<X(n)<1$ ]. The field-ionization signal $I_{j}$ caused by the $j$ th pulse would be given, in this case, by

$$
I_{j}=N_{0} X(n)[1-X(n)]^{j-1},
$$

where $N_{0}$ represents the total population of Rydberg states prepared optically.

To test the validity of Eq. (1), the effect of a sequence of nine equivalent pulsed fields $(10 \mathrm{~V} / \mathrm{cm}$, rise time 70 ns, interval $550 \mathrm{~ns}$ ) on the Rydberg states below the ${ }^{2} P_{3 / 2}$ threshold, has been investigated. Figures 3(a)-3(f)
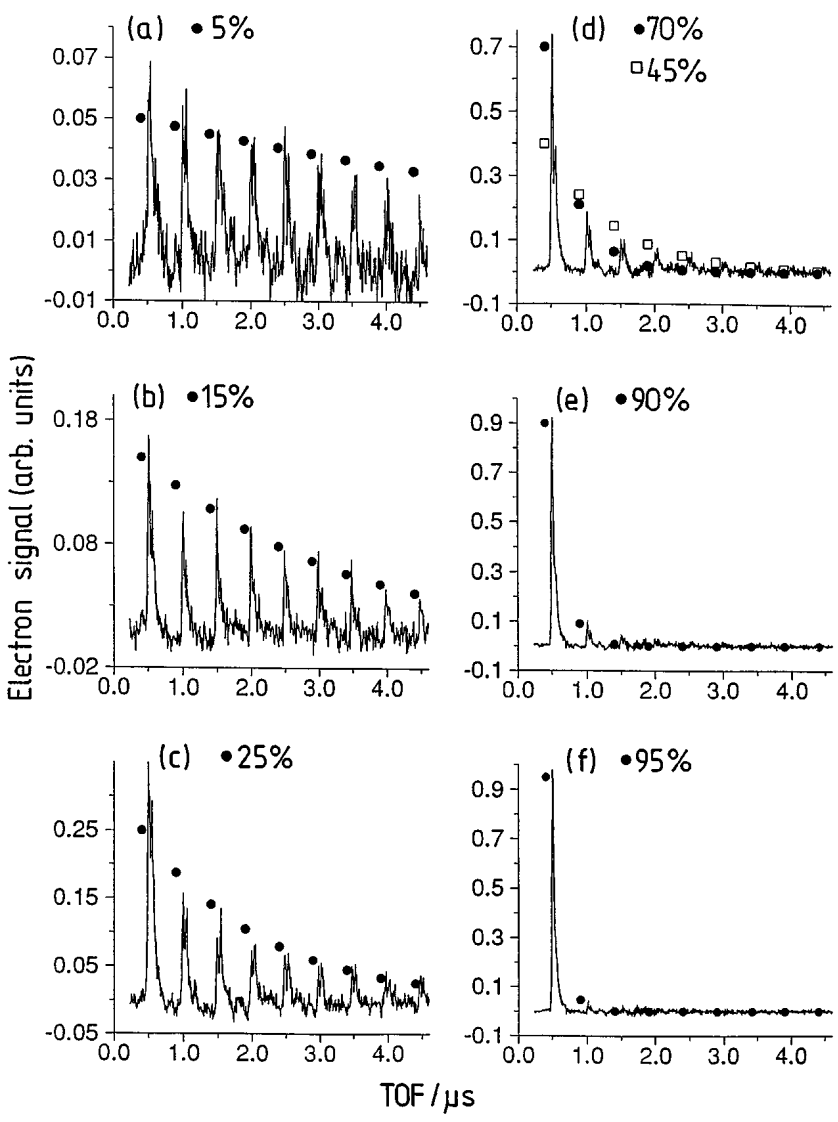

FIG. 3. Electron TOF profiles following PFI of Rydberg states at energies (a) $-18.5 \mathrm{~cm}^{-1}$, (b) $-15.8 \mathrm{~cm}^{-1}$, (c) $-14.3 \mathrm{~cm}^{-1}$, (d) $-13.0 \mathrm{~cm}^{-1}$, (e) $-11.8 \mathrm{~cm}^{-1}$, and (f) $-10.3 \mathrm{~cm}^{-1}$, below the ${ }^{2} P_{3 / 2}$ threshold obtained with a sequence of nine equivalent pulsed fields $(10 \mathrm{~V} / \mathrm{cm}$, rise time $70 \mathrm{~ns}$, interval $550 \mathrm{~ns}$ ). The full circles [and squares in (d)] represent predictions of sequential field-ionization signals using Eq. (1), with the single-pulse ionization efficiencies indicated. 


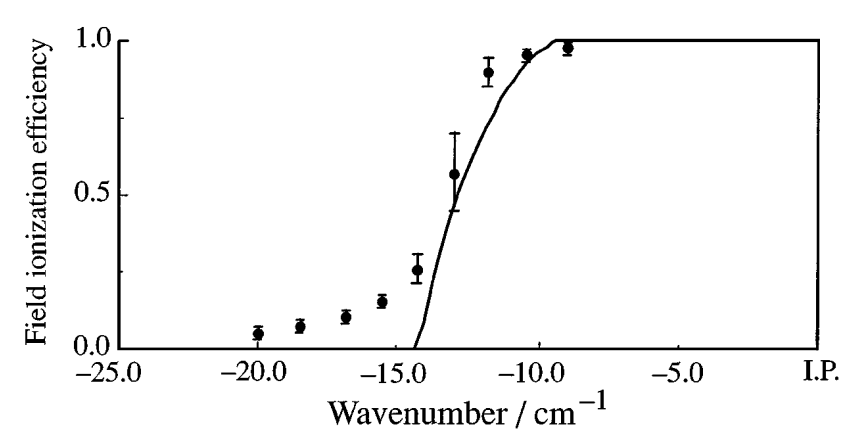

FIG. 4. Comparison between field-ionization efficiencies measured experimentally with a nine pulse sequence (circles) and diabatic field-ionization efficiencies calculated using hydrogenic field-ionization rates [12] (full line).

compare the electron TOF profiles with the predictions of Eq. (1) (full circles or squares in the figure) for different excitation energies. At the lowest [Fig. 3(a) and 3(b)] and highest [Fig. 3(f)] energies, the experimental profiles are accounted for quantitatively by Eq. (1) with $X=0.05,0.15$, and 0.95 , respectively. At intermediate energies, the experimental results cannot be fitted perfectly by Eq. (1): The ionization efficiency is highest for the first pulse $[X \sim 0.7$ in Fig. 3(d) $]$ and decreases progressively at subsequent pulses $[X \sim 0.45$ for the latest peaks in Fig. 3(d)].

The energy-dependent field-ionization efficiencies determined from fits to the profiles of Fig. 3 are compared in Fig. 4 with those derived using expressions for diabatic field-ionization rates [Eq. (6) of Ref. [12]]. Apart from the low frequency tail exhibited by the experimental results, which is caused by nondiabatic field ionization, the agreement between theory and experiment is good. This comparison also provides an explanation for the deviations from the predictions of Eq. (1) observed in Figs. 3(c)3(e). The bandwidth of the exciting radiation $\left(1.5 \mathrm{~cm}^{-1}\right)$ leads to the simultaneous excitation of several $n$ manifolds, whereas Eq. (1) applies to a single value of $n$. The behavior of the prepared Rydberg population therefore necessarily exhibits a range of field-ionization rates and yields. Deviations are expected to be particularly pronounced, and are indeed observed (see Figs. 3 and 4) in the region where the field-ionization efficiency changes very rapidly with energy, provided that the randomization occurs only among states of a single Stark manifold of given $n$, but not between different Stark manifolds. Explicit consideration of this effect leads to the conclusion that the experimental data are consistent with a statistical redistribution of the Rydberg population among all accessible Stark states in a given manifold in the interval between two pulses and that Eq. (1) can be successfully used to predict how many pulsed electric fields are necessary to field ionize a complete Rydberg state population.

The complete memory loss of $k$ is surprising, in that the surviving Rydberg atoms must exist in high- $m$ states and only undergo diabatic crossings, especially near the ${ }^{2} P_{1 / 2}$ limit. One explanation would be that the $m$ mixing, responsible for stabilization of these states, occurs not only at the time of excitation but also when the field returns near zero, where all $(m, k)$ states are near degenerate. This converts some of the population back to low- $m$ states, facilitating the $k$-mixing.

We are grateful for financial support from EPSRC, the Paul Fund, and St John's College, Oxford.

[1] T.F. Gallagher, Rydberg Atoms (Cambridge University Press, Cambridge, 1994).

[2] Rydberg States of Atoms and Molecules, edited by R.F. Stebbings and F. B. Dunning (Cambridge University Press, New York, 1983).

[3] W. A. Chupka, J. Chem. Phys. 98, 4520 (1993).

[4] D. Bahatt, U. Even, and R. D. Levine, J. Chem. Phys. 98, 1744 (1993).

[5] F. Merkt and T.P. Softley, in High-Resolution Laser Photoionization and Photoelectron Studies, edited by I. Powis, T. Baer, and C. Y. Ng (Wiley, New York, 1995).

[6] F. Merkt and R. N. Zare, J. Chem. Phys. 101, 3495 (1994).

[7] F.H. Kellert, T.H. Jeys, G. B. Macmillan, K. A. Smith, F. B. Dunning, and R. F. Stebbings, Phys. Rev. A 23, 1127 (1981).

[8] M. J. J. Vrakking and Y.T. Lee, J. Chem. Phys. 102, 8833 (1995).

[9] F. Merkt, J. Chem. Phys. 100, 2623 (1994).

[10] J. R. Rubbmark, M. M. Kash, M.G. Littman, and D. Kleppner, Phys. Rev. A 23, 3107 (1981).

[11] C. Y. Lee, J. M. Heffena, T.F. Gallagher, and C. W. S. Conover, Phys. Rev. A 46, 7048 (1992).

[12] R. J. Damburg and V. V. Kolosov, J. Phys. B 12, 2637 (1979). 